Discrete Comput Geom 36:283-311 (2006)

DOI: $10.1007 / \mathrm{s} 00454-006-1231-5$

\title{
A Linear Time Algorithm for Constructing Maximally Symmetric Straight Line Drawings of Triconnected Planar Graphs*
}

\author{
Seok-Hee Hong, ${ }^{1}$ Brendan McKay, ${ }^{2}$ and Peter Eades ${ }^{1}$ \\ ${ }^{1}$ School of Information Technologies, University of Sydney, \\ Sydney, NSW 2006, Australia \\ \{shhong,peter\}@cs.usyd.edu.au \\ and \\ National ICT Australia, \\ Eveleigh, NSW 1430, Australia \\ ${ }^{2}$ Department of Computer Science, Australian National University, \\ Canberra, ACT 0200, Australia \\ bdm@cs.anu.edu.au
}

\begin{abstract}
Symmetry is one of the most important aesthetic criteria in graph drawing because it reveals structure in the graph. To draw graphs symmetrically, we employ two steps. The first step is to find appropriate automorphisms. The second step is to draw the graph to display the automorphisms. Our aim in this paper is to construct maximally symmetric straight line drawings of triconnected planar graphs in linear time. Previously known algorithms run in quadratic time. We show that an algorithm of Fontet can be used to find an embedding in the plane with the maximum number of symmetries, and present a new algorithm for finding a straight line drawing that achieves that maximum. Both algorithms run in linear time.
\end{abstract}

\section{Introduction}

Drawings of graphs in graph theory textbooks are often symmetric [5]. Symmetry clearly reveals structure, especially algebraic structure, in the graph. Further, HCI experiments have shown that symmetry is an important consideration in the understanding of drawings of graphs that arise in applications such as Software Engineering [30].

The problem of determining whether a given graph can be drawn symmetrically is NP-complete in general [25], [27].

* An extended abstract of this paper appeared in [22]. This research has been supported by a grant from the Australian Research Council. This paper was partially written when the first author was visiting the Australian National University. 
Heuristics for constructing symmetric drawings of general graphs have been suggested by Lipton et al. [24] and de Fraysseix [11]. Exact algorithms are devised based on the Branch and Cut approach by Buchheim and Junger [6] and a group-theoretic approach by Abelson et al. [1]. A group-theoretic characterization of symmetric drawing was given by Eades and Lin [9].

Linear time algorithms are available for restricted classes of graphs by Manning and Atallah [28], [29], including trees [28], outerplanar graphs [29], and plane graphs [27]. A linear time algorithm is available for constructing maximally symmetric drawings of series-parallel digraphs [20], [21].

Recently, symmetric drawing in three dimensions has been considered [4], [12][14]. The problem of determining whether a graph can be drawn symmetrically in three dimensions is NP-complete [12]. Linear time algorithms are available for constructing maximally symmetric drawings of trees [13], [16] and series-parallel digraphs in three dimensions [14] by Hong and Eades. Bern and Eppstein present a method for constructing a symmetric drawing on a sphere [4].

To construct a drawing that displays as much symmetry as possible, we use two steps. The first step, called the symmetry finding step, is to find a "displayable" automorphism group of maximum size. The second step, called the drawing step, is to construct a drawing that displays these automorphisms.

A linear time symmetry finding algorithm for embedded planar graphs, proposed by Manning [27], can be used to find displayable automorphisms for triconnected planar graphs in quadratic time: one can use Manning's algorithm on each of the $O(n)$ planar embeddings formed by choosing an outside face. Section 3 of this paper gives a linear time algorithm for finding a maximum size group of displayable symmetries in triconnected planar graphs.

To construct straight line symmetric drawings of triconnected planar graphs, one can use the algorithm by Tutte [33]. However, this runs in $O\left(n^{1.5}\right)$ time at best. Several algorithms give straight line drawings in linear time but without symmetry (see [8]). Section 4 of this paper gives a linear time algorithm for constructing straight line symmetric drawings of triconnected planar graphs.

The following theorem summarizes our main result.

Theorem 1. There is a linear time algorithm that constructs maximally symmetric planar drawings of triconnected planar graphs, with straight line edges.

The proof of Theorem 1 consists of two algorithms: an algorithm in Section 3 for finding the maximum number of displayable symmetries and a symmetric drawing algorithm in Section 4. Both algorithms run in linear time.

Firstly, however, we present the necessary background: symmetries and planar automorphisms.

\section{Symmetries and Planar Automorphisms}

There are two types of symmetry in two-dimensional drawings: rotational symmetry and axial (reflectional) symmetry. A rotational symmetry is a rotation about a point and an axial symmetry is a reflection about an axis. 


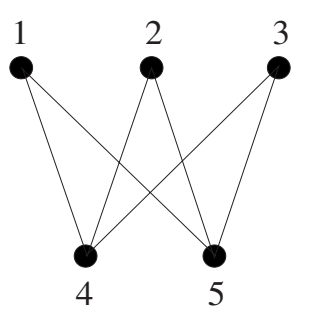

(a)

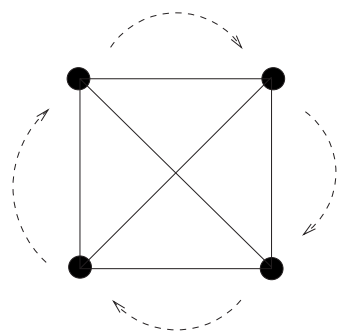

(b)

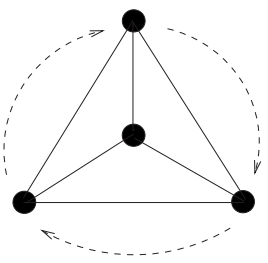

(c)

Fig. 1. (a) The automorphism $(1,2,3)(4,5)$ is not geometric. (b) The automorphism that cycles all four vertices is geometric but not planar. (c) A drawing of $K_{4}$ displaying a dihedral group of size 6 .

An automorphism of a graph is a permutation of the vertex set that preserves adjacency. Symmetry in graph drawing is closely related to automorphisms of graphs: a symmetry of a graph drawing induces an automorphism of the graph. In this case we say that the drawing displays the automorphism. If an automorphism is displayed as a symmetry in a drawing of the graph, then it is a displayable or geometric automorphism. Note that not every automorphism is geometric; see Fig. 1(a). This formal model for symmetric drawing in two dimensions was introduced by a number of authors [9], [19], [20], [27][29].

An automorphism of a graph $G$ is a planar automorphism if there is a planar drawing of $G$ which displays it. Note that not every geometric automorphism is planar; see Fig. 1(b).

Note that the product of two planar automorphisms is not necessarily planar, because they may be displayed by different drawings. For example, consider two rotational symmetries of $K_{4}$ which have different fixed points, each displayed in a drawing as in Fig. 1(c). Their product, a pair of transpositions, is an axial symmetry, and can only be displayed in a nonplanar drawing such as in Fig. 1(b). A subgroup $A$ of the automorphism group of a graph is a planar automorphism group if there is a single planar drawing of the graph that displays every element of $A$ (see Fig. 1(c)). The first task in this paper is to find a maximum size planar automorphism group for a triconnected planar graph.

If $g_{1}, g_{2}, \ldots, g_{\ell}$ are automorphisms, then the group generated by $g_{1}, g_{2}, \ldots, g_{\ell}$ is denoted by $\left\langle g_{1}, g_{2}, \ldots, g_{\ell}\right\rangle$. This group consists of all permutations formed from products of elements of $\left\{g_{1}, g_{2}, \ldots, g_{\ell}\right\}$. We say that $g_{1}, g_{2}, \ldots, g_{\ell}$ are generators of $\left\langle g_{1}, g_{2}, \ldots, g_{\ell}\right\rangle$.

The order of an automorphism $\alpha$ is the smallest positive integer $k$ such that $\alpha^{k}$ equals the identity. The size (or order) of an automorphism group is the number of elements of the group. A group-theoretic characterization of geometric automorphisms follows [9], [23].

Theorem 2 [9], [23]. A nontrivial subgroup A of the automorphism group of a graph $G=(V, E)$ is geometric if and only if the permutation group on $V$ defined by $A$ is one of the following types:

1. $A=\langle\alpha\rangle$ where $\alpha$ has order 2 ; or

2. $A=\langle\rho\rangle$ where $\rho$ is a rotational permutation; or 
3. $A=\langle\rho, \alpha\rangle$ such that:

(a) $\rho$ is a rotational permutation and $\alpha$ has order 2 ,

(b) $\langle\rho\rangle \cap\langle\alpha\rangle$ is trivial and

(c) $\alpha \rho=\rho^{-1} \alpha$.

One can deduce that a nontrivial planar automorphism group is one of three kinds: (1) a group of size 2 induced by an axial symmetry; (2) a cyclic group of size $k$ induced by a rotational symmetry of order $k$; (3) a dihedral group of size $2 k$ induced by a rotational symmetry of order $k$ and an axial symmetry.

\section{Finding a Planar Automorphism Group of Maximum Size}

In this section we show that there is a linear time algorithm for finding a planar automorphism group of maximum size of triconnected planar graphs. The output of the algorithm is a set of generators for the group, explicitly represented as permutations on the vertices. Further, the generators have a minimality property (described later) that is useful for the drawing algorithm in Section 4.

The algorithm relies on two fundamental theorems, as follows.

Theorem 3 [3], [26]. Every triconnected planar graph $G$ can be realized as the 1skeleton of a convex polytope $P$ in $\mathbb{R}^{3}$ such that all automorphisms of $G$ are induced by isometries of $P$.

To state the next theorem, we need some definitions from group theory: suppose that $A$ is a group acting on a set $X$. The stabilizer of $x \in X$, denoted by $\operatorname{stab}_{A}(x)$, is $\{g \in A \mid g(x)=x\}$, and the orbit of $x$, denoted by orbit $_{A}(x)$, is $\{g(x) \mid g \in A\}$.

We say that $g \in A$ fixes $x \in X$ if $g(x)=x$; if $g$ fixes $x$ for every $g \in A$ then $A$ fixes $x$. If $X^{\prime} \subseteq X$ and $g\left(x^{\prime}\right) \in X^{\prime}$ for all $x^{\prime} \in X^{\prime}$ then $g$ fixes $X^{\prime}$; if $g$ fixes $X^{\prime}$ for every $g \in A$ then $A$ fixes $X^{\prime}$. It is useful to note that the term fix when applied to a set is taken setwise, rather than elementwise; for example, if $g$ fixes the edge $(u, v)$ then it fixes the set $\{u, v\}$; it does not necessarily fix $u$ or $v$ (it may swap $u$ and $v$ ).

Theorem 4 (Orbit-Stabilizer Theorem [2]). Suppose that $A$ is a group acting on a set $X$ and let $x \in X$. Then $|A|=\mid$ orbit $_{A}(x)|\times|$ stab $_{A}(x) \mid$.

We now characterize planar automorphisms.

Lemma 1. Let $G$ be a triconnected planar graph. An automorphism of $G$ is a planar automorphism if and only if it fixes a face of $G$.

Proof. Necessity comes from the fact that the outside face must be fixed by any symmetry. Sufficiency comes from Theorem 3, using a projection about a face from the polyhedron to the plane. 
Based on Lemma 1, we now describe our algorithm for finding a maximum size planar automorphism group of a triconnected planar graph. The overall algorithm can be described as follows:

\section{Algorithm Find_Max_PAG}

1. Find a plane embedding which has a maximum size planar automorphism group.

2. Perform "star triangulation" (defined below) for the given embedding.

3. Find the generators of the planar automorphism group of the new embedding.

The first step uses two applications of an algorithm of Fontet [10]. This algorithm takes a triconnected planar graph as input and outputs the orbits of vertices induced by the (full) automorphism group of the graph. It runs in linear time.

The first step in our algorithm is described in the following theorem.

Theorem 5. Fontet's algorithm can be used to find an embedding of a triconnected graph such that the corresponding planar automorphism group is maximized. This takes linear time.

Proof. Let $G$ be a triconnected planar graph. Motivated by Lemma 1, we take the dual graph of $G^{*}$ of $G$ and compute the orbits of $G^{*}$ using Fontet's algorithm. Then we choose a vertex $f$ of $G^{*}$ so that the orbit of $f$ has minimum size; by Theorem 4, the stabilizer of $f$ has maximum size. Taking any face in the orbit of $f$ as the outside face of the plane embedding of $G$, we have a maximum size planar automorphism group.

Once we have chosen the outside face, and thus the planar embedding, we triangulate each internal face by inserting a new vertex in the face and joining it to each vertex of the face. This process is called star triangulation and is illustrated in Fig. 2. Clearly it takes only linear time. This step is not strictly required for Algorithm Find_Max_PAG, but it simplifies the algorithm slightly, and we need the star triangulation for the next section.

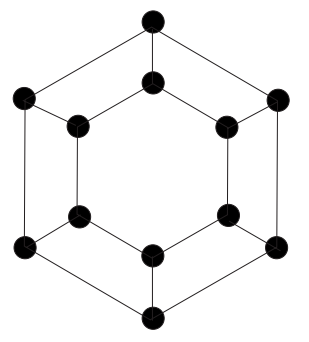

(a)

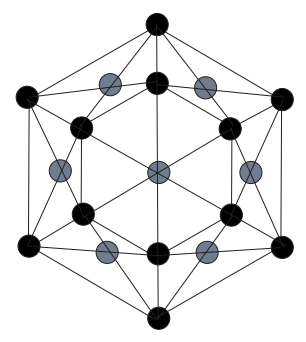

(b)

Fig. 2. Example of star triangulation. 
Star triangulation has the useful property of preserving the planar automorphism group:

Lemma 2. Let $G$ be a triconnected plane graph and let $G^{\prime}$ be the graph obtained from $G$ by star triangulation. Suppose that $A$ is the planar automorphism group of $G$. Then $G^{\prime}$ has planar automorphism group $A^{\prime}$ isomorphic to A, such that the action of $A^{\prime}$ on the vertices of $G$ is precisely $A$.

Proof. Since $A$ maps faces to faces, it is clear that one can extend $A$ to a permutation group $A^{\prime}$ acting on the vertices of $G^{\prime}$. To show that $A^{\prime}$ is isomorphic to $A$, we need to prove that planar automorphisms of $G^{\prime}$ cannot map one of the new vertices onto an old vertex (that is, a vertex of $G$ ). We do this by showing that the new vertices are distinguishable combinatorially. Form a graph $G^{\prime \prime}$ from $G^{\prime}$ by adding a new vertex $v$ to the outside face and joining $v$ to all vertices on the outside face. Say that two vertices of $G^{\prime \prime}$ are opposed if they are the vertices opposite an edge in two faces that share that edge. Then each vertex $w$ of $G^{\prime \prime}$ is a new vertex (not in $G$ ) if and only if there is a sequence $v=v_{0}, v_{1}, \ldots, v_{m}=w$ such that $v_{i-1}$ and $v_{i}$ are opposed for $1 \leq i \leq m$.

We now explain the third step of Algorithm Find_Max_PAG. Our aim is to find the planar automorphism group for our star-triangulated planar graph. However, an explicit representation of the planar automorphism group may take more than linear space. For a more compact representation, we find generators.

Further, in the case that the group is cyclic, we need a "minimality" property of its generator $\rho$, defined as follows: $\rho$ is minimal if $\rho(x)$ is the closest vertex to $x$ out of the $\rho^{i}(x)$ along the outside face. The minimality property is important when the generators are used in the drawing algorithm in Section 4.

Before we describe the generator-finding method, we define some terminology. A flag of an embedded graph is a triple $(v, w, f)$, where $v$ and $w$ are adjacent vertices and $f$ is a face that has the edge $(v, w)$ on its boundary.

The following lemma is helpful in our algorithm.

Lemma 3. Let $G$ be a connected graph embedded on the sphere. Let $F=(v, w, f)$ and $F^{\prime}=\left(v^{\prime}, w^{\prime}, f^{\prime}\right)$ be flags of $G$. Then there is at most one automorphism of $G$ that both preserves the embedding of $G$ and maps $F$ onto $F^{\prime}$. Moreover, there is a linear time algorithm which finds that automorphism, or determines that it does not exist.

Proof. This lemma and its proof are well known, but we provide an outline of the proof for completeness.

Perform a depth-first search of $G$ starting with $v$. The neighbours of $v$ must be scanned starting with $w$, in the order determined by $F$ : clockwise order if $f$ is on the right of $v w$ and anticlockwise order otherwise. In the case of vertices other than $v$, the neighbours must be scanned in the same cyclic order as before, starting with the parent vertex. This clearly defines a unique order of visiting for the vertices and edges of $G$, depending only on the combinatorial structure of $G$, its embedding and the starting flag. Create a copy of $G$ in which all the vertices and edges are labelled in the order that they are visited. 
Now perform the same operation starting at the second flag $F^{\prime}$. This produces a second labelled copy of $G$. If there is an automorphism of $G$ that preserves the embedding and maps $F$ onto $F^{\prime}$, the two labelled graphs are the same (same adjacencies and same cyclic order of edges at each vertex) and the correspondence between the two copies gives the automorphism. Conversely, if the labelled graphs are not the same, there is no such automorphism.

We now give the main algorithm for finding generators of the planar automorphism group which has maximum size.

Theorem 6. Let $G$ be a triconnected graph embedded in the plane with prescribed outside face and every internal face a triangle. Let $A$ be the group of automorphisms of $G$ which preserve the outside face. Then there is a linear time algorithm for finding generators for A. In the case where the group is cyclic, the generator is minimal.

Proof. Let $f$ be the outside face of $G$, and let $v_{0}, v_{1}, \ldots, v_{m-1}$ be its vertices in clockwise order. In the following, subscripts of $v$ are interpreted modulo $m$.

If $m>3$, then the outside face of $G$ is fixed by all automorphisms since it is the only face of that size. In that case, application of Fontet's algorithm to $G$ gives the orbits of $A$ on the vertices of $G$.

If $m=3$, the automorphisms of $G$ on the sphere might map the outside face of $G$ onto another face. Then a simple modification of $G$ by adding a new outside face can be used to find the orbits. More specifically, modify $G$ temporarily as follows: Draw a hexagon surrounding $G$ in the plane, with vertices $w_{0}, w_{1}, \ldots, w_{5}$ in clockwise order. Insert the edges $v_{0} w_{0}, v_{0} w_{1}, v_{0} w_{2}, v_{1} w_{2}, v_{1} w_{3}, v_{1} w_{4}, v_{2} w_{4}, v_{2} w_{5}$, and $v_{2} w_{0}$. The extended graph has a new outside face of size 6 and all other faces are triangles. Now apply Fontet's algorithm to the extended graph and afterwards remove the temporary vertices and edges. The result is the orbits of $A$ on the vertices of $G$.

Next we show how to find generators from the orbits. If $v_{0}, v_{1}, \ldots, v_{m-1}$ are each fixed by $A$, then $A$ is trivial. Otherwise, let $v_{i}, v_{j}, v_{k}$ be three consecutive vertices in the same nontrivial orbit of $A$, where $j-i$ is as small as possible and $v_{k}$ is the same as $v_{i}$ if the orbit has size 2. Apply Lemma 3 to find three possible automorphisms or prove that they do not exist:

- $\alpha$ is the automorphism mapping the flag $\left(v_{i}, v_{i+1}, f\right)$ onto the flag $\left(v_{j}, v_{j-1}, f\right)$, if that automorphism exists. (That is, a reflection that exchanges $v_{i}$ and $v_{j}$.)

- $\rho_{1}$ is the automorphism mapping the flag $\left(v_{i}, v_{i+1}, f\right)$ onto the flag $\left(v_{j}, v_{j+1}, f\right)$, if that automorphism exists. (That is, a rotation by $j-i$ positions.)

- $\rho_{2}$ does not exist in the case that $v_{k}=v_{i}$. Otherwise, $\rho_{2}$ is the automorphism mapping the flag $\left(v_{i}, v_{i+1}, f\right)$ onto the flag $\left(v_{k}, v_{k+1}, f\right)$, if that automorphism exists. (That is, a rotation by $k-i$ positions.)

Now we can identify $A$ :

- If $\alpha$ does not exist, then $\rho_{1}$ exists and $A$ is a cyclic group of size $m /(j-i)$ generated by the rotation $\rho_{1}$. 
- If $\alpha$ exists but neither $\rho_{1}$ nor $\rho_{2}$ exists, then $A$ is the group of size 2 generated by the reflection $\alpha$.

- If $\alpha$ and $\rho_{1}$ exist, then $A$ is the dihedral group of size $2 m /(j-i)$ generated by the reflection $\alpha$ and the rotation $\rho_{1}$.

- Otherwise, $\alpha$ and $\rho_{2}$ exist, and $A$ is the dihedral group of size $2 m /(k-i)$ generated by the reflection $\alpha$ and the rotation $\rho_{2}$.

It is easy to check the minimality of $\rho_{1}$ and $\rho_{2}$. This completes the proof.

We now summarize the main result of this section.

Theorem 7. Algorithm Find_Max_PAG computes generators of the maximum size planar automorphism group of a triconnected planar graph in linear time.

\section{The Symmetric Drawing Algorithm}

In this section we present a linear time algorithm for finding a straight line planar drawing of a triconnected planar graph such that maximum number of planar automorphisms are displayed. From Section 3 we can assume that we have generators for a maximum planar automorphism group and an embedding that can display it. Moreover, the interior faces have been star-triangulated. In fact we prove the following theorem.

Theorem 8. Given a triconnected planar graph $G$ and generators of a planar automorphism group $A$ of $G$, there is a linear time algorithm that outputs a straight line drawing of $G$ that displays $A$.

An overview of the algorithm is in Section 4.3. Its proof, as well as several subroutines used in the algorithm, is given in Sections 4.4-4.6. However, first we review two classical algorithms:

- The algorithm of Tutte [32], [33], which gives symmetric drawings, although it has a higher time complexity, in Section 4.1.

- The algorithm of Chiba et al. [7], which takes linear time but does not give symmetric drawings, in Section 4.2.

\subsection{The Tutte Algorithm}

Tutte [32], [33] (see [8]) gave a simple graph drawing algorithm. The algorithm takes a triconnected planar graph $G$ and an $m$-vertex face $F$ of $G$, draws $F$ as a regular $m$-gon, and places each vertex of $G-F$ at the barycenter of its graph-theoretic neighbours. The edges of $G$ are drawn as straight lines. Tutte showed that the resulting drawing is planar and has convex faces.

The algorithm can be implemented by solving a system of linear equations; the sparsity of the equations allows an implementation in time $O\left(n^{1.5}\right)$. 
The following theorem is folklore; it can be proven simply from the equations (see [23] for details).

Theorem 9. Given a triconnected planar graph $G$ and a face $F$ of $G$, if $\tau$ is a planar automorphism of $G$ that fixes $F$ then the Tutte drawing of $G$ displays $\tau$.

Although we do not use Tutte's algorithm in our symmetry drawing algorithm, Theorem 9 is important in the proof of some results in Sections 4.5 and 4.6 below that ensure the correctness of our algorithm.

\subsection{The Algorithm of Chiba et al.}

Chiba et al. [7] gave a linear time algorithm for drawing a biconnected plane graph with straight line edges and convex faces.

We need some terminology. A vertex of a polygon is an apex if the interior angle is less than $\pi$. A polygon is weakly convex if all interior angles are at most $\pi$.

The input to their algorithm is a biconnected plane graph with given outside face and a straight line drawing of this face as a weakly convex polygon; not every vertex of the outside face needs to be at an apex of the polygon. The output of their algorithm is a straight line drawing of the biconnected plane graph with the outside face drawn as the input convex polygon, if this is possible. The conditions under which such a drawing exists is a classical theorem of Thomassen [31].

Theorem 10 [31]. Let $G$ be a biconnected plane graph with outside facial cycle $S$, and let $S^{*}$ be a drawing of $S$ as a convex polygon. Let $P_{1}, P_{2}, \ldots, P_{k}$ be the paths in $S$, each corresponding to a side of $S^{*}$. Then $S^{*}$ can be extended to a convex drawing of $G$ if and only if the following three conditions hold:

1. For each vertex $v$ of $G-V(S)$ having degree at least 3 in $G$, there are three paths disjoint except at $v$, each joining $v$ and a vertex of $S$.

2. The graph $G-V(S)$ has no connected component $C$ such that all the vertices on $S$ adjacent to vertices in $C$ lie on a single path $P_{i}$; and no two vertices in each $P_{i}$ are joined by an edge not in $S$.

3. Any cycle of $G$ which has no edge in common with $S$ has at least three vertices of degree at least 3 in $G$.

We briefly outline the algorithm of Chiba et al. which effectively implements Thomassen's theorem. The algorithm reduces the convex drawing of $G$ to those of several subgraphs of $G$ as follows:

Algorithm CYN ( $\left.G, S, S^{*}\right)$

1. Delete from $G$ an arbitrary vertex $v$ at an apex of $S^{*}$ together with edges incident to $v$.

2. Divide the resulting graph $G^{\prime}=G-v$ into the biconnected components $B_{1}, B_{2}, \ldots, B_{p}, p \geq 1$. 


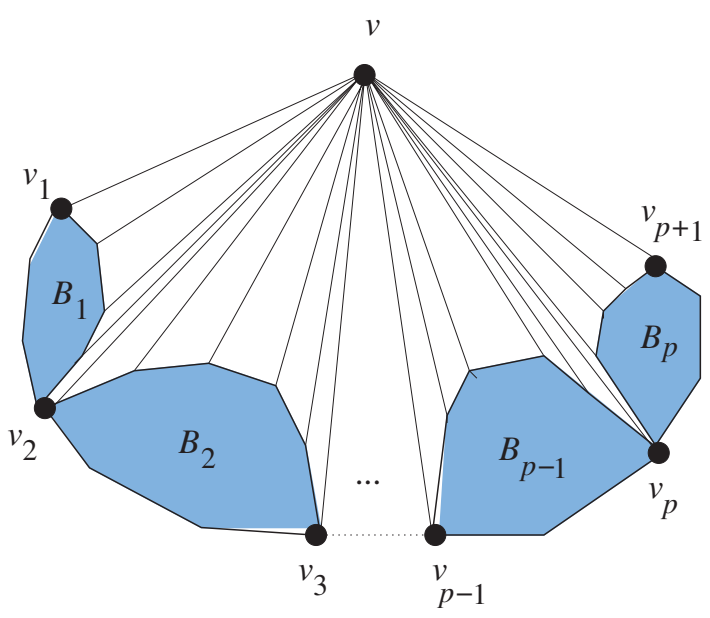

Fig. 3. The algorithm of Chiba et al.

3. Determine a convex polygon $S_{i}^{*}$ of the outside facial cycle $S_{i}$ of each $B_{i}$ so that $B_{i}$ with $S_{i}^{*}$ satisfy the condition in Theorem 10 .

4. Recursively apply the algorithm to each $B_{i}$ with $S_{i}^{*}$ to determine the positions of vertices not in $S_{i}$.

This is illustrated in Fig. 3. For details, see [7].

Our applications of Theorem 10 will be to subgraphs of a triconnected planar graph defined by a cycle and its interior. In that case Thomassen's conditions can be simplified.

Suppose that $P$ is a path in a graph $G$; a chord for $P$ in $G$ is an edge of $G$ not in $P$, but whose endpoints are in $P$.

Corollary 1. Let $G$ be a triconnected plane graph and let $S$ be a cycle of $G$. Let W be the graph consisting of $S$ and its interior. Let $S^{*}$ be a drawing of $S$ as a convex $k$-gon (where $S$ might have more than $k$ vertices). Let $P_{1}, P_{2}, \ldots, P_{k}$ be the paths in $S$ corresponding to the sides of $S^{*}$. Then $S^{*}$ is extendible to a straight line planar drawing of $W$ if and only if no path $P_{i}$ has a chord in $W$.

Proof. We can assume that the interior faces of $W$ are triangles, since otherwise we can star triangulate them, draw the graph using straight lines, and then undo the star triangulation.

We need to show that Thomassen's three conditions are met. Condition 1 is a standard implication of the triconnectivity of $G$, and Condition 3 follows just from the observation that the internal vertices have degree at least 3.

To prove the first part of Condition 2, suppose that $C$ is a connected component of $W-S$ which is adjacent to $P_{i}$ but not to any other of $P_{1}, \ldots, P_{k}$. Let $u$ and $v$ be the first and last vertices on $P_{i}$ which are adjacent to $C$. If $u=v$, or $u$ and $v$ are adjacent on $P_{i}$, then $\{u, v\}$ is a cut (separation pair) in $G$, which is impossible as $G$ is triconnected. 
Otherwise, there is an interior face containing $u$ and $v$ and so $u$ and $v$ are adjacent, contrary to our hypothesis (since the internal faces are triangles).

The second part of Condition 2 is just the condition that we are imposing.

\subsection{Overview of the Drawing Algorithm}

A major characteristic of symmetric drawings is the repetition of congruent (isomorphic) drawings of isomorphic subgraphs. To exploit this property, we compute a drawing for a subgraph and then use copies of it to draw the whole graph.

The input of the drawing algorithm is a triconnected planar graph with fixed planar embedding, that is, specified with outside face. The embedding is the one which maximizes the number of symmetries, which can be found using the algorithm in Section 3.

As stated in Section 2, the planar automorphism group has one of the following three types:

1. a group of size 2 generated by an axial symmetry;

2. a cyclic group of size $k$ generated by a rotational symmetry of order $k$;

3. a dihedral group of size $2 k$ generated by a rotational symmetry of order $k(k>1)$ and an axial symmetry. In this case there are $k$ axial symmetries.

Our drawing algorithm will take a different approach for each of the above cases.

Note that after the star triangulation, there is either a vertex or an edge which is fixed by the planar automorphism group. This is described in the following lemma.

Lemma 4. Let $G$ be a triconnected plane graph and let $G^{\prime}$ be the graph obtained from $G$ by star triangulation. Suppose that $A^{\prime}$ is the planar automorphism group of $G^{\prime}$ of size $\ell$. If $\ell>2$, then there is a central vertex in $G^{\prime}$ which is fixed by $A^{\prime}$. If $\ell=2$, then either a central vertex or a central edge is fixed by $A^{\prime}$.

The overall symmetric drawing algorithm can be described as follows:

\section{Algorithm Symmetric_Drawing}

Based on the type of the planar automorphism group, execute one of the following algorithms (whose definition will follow):

1. Cyclic.

2. One_Axial.

3. Dihedral.

The remainder of this section consists of providing a drawing algorithm for each of the three kinds of planar automorphism group.

Algorithms Cyclic and Dihedral, described in Section 4.4 and Section 4.6 respectively, follow a similar pattern. First we find a subgraph. Then we draw the subgraph using the algorithm of Chiba et al. [7] as a subroutine. The last step is to replicate the drawing; that is, merge copies of the drawings of the subgraphs to construct a drawing of the whole graph. 
Algorithm One_Axial, described in Section 4.5, uses a variation of CYN.

Now we consider each of the three parts of Algorithm Symmetric_Drawing.

\subsection{The Cyclic Case}

In this section we describe how to display $k$ rotational symmetries. This is the simplest case, and the general principle is used in the dihedral case in Section 4.6.

Firstly suppose that $k \geq 3$; from Lemma 4 there is a central vertex $c$. Our symmetric drawing algorithm consists of three steps.

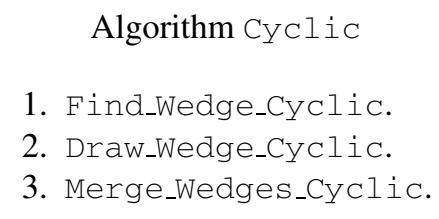

We now describe each step in turn. The first step is to find a subgraph $W$, called a wedge, as follows:

\section{Algorithm Find_Wedge_Cyclic}

1. Find the central vertex $c$.

2. Find a shortest path $P_{1}$ from $c$ to a vertex $v_{1}$ on the outside face. This can be done in linear time by breadth-first search.

3. Find the path $P_{2}$ which is a mapping of $P_{1}$ under a minimal generator of the rotation.

4. Find the induced subgraph of $G$ enclosed by the cycle formed from $P_{1}, P_{2}$, and a path $P_{0}$ along the outside face from $v_{1}$ to $v_{2}$ (including the cycle). This is the wedge $W$.

An example of a wedge $W$ is illustrated in Fig. 4.

It is clear that Algorithm Find_Wedge_Cyclic runs in linear time.

The second step, Draw_Wedge_Cyclic, constructs a drawing $D$ of the wedge $W$ using Algorithm CYN, such that $P_{1}, P_{2}$, and $P_{0}$ are drawn as straight lines. This is possible by the next lemma.

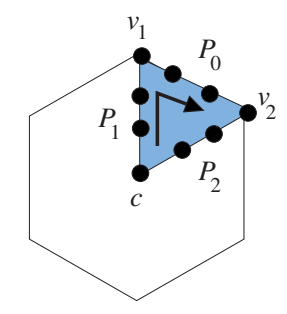

Fig. 4. Example of a wedge. 


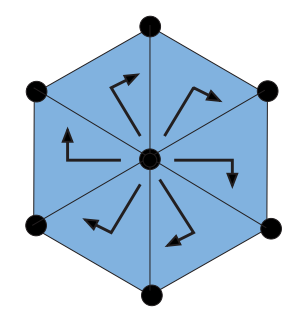

Fig. 5. Example of merging step.

Lemma 5. Suppose that $W$ is the wedge computed by Algorithm Find_Wedge_Cyclic, $S$ is the outside face of $W$ and $S^{*}$ is the drawing of $S$ using straight lines for $P_{1}, P_{2}$, and $P_{0}$, as in Fig. 4. Then $W$ and $S^{*}$ satisfy the conditions of Corollary 1.

Proof. Since $k \geq 3$, the fixed central vertex $c$ common to $P_{1}$ and $P_{2}$ is an apex. Thus the only straight lines in $S^{*}$ of the drawing are the drawings of $P_{1}$ and $P_{2}$. These paths cannot have chords, as they are the shortest paths in $G$.

The last step, Merge_Wedges_Cyclic, constructs a drawing of the whole graph $G$ by replicating the drawing $D$ of $W, k$ times. Note that this merge step relies on the fact that $P_{1}$ and $P_{2}$ are drawn as straight lines. This is illustrated in Fig. 5.

Now consider the case that $k=2$. From Lemma 4 there may be a central vertex or a central edge fixed by the planar automorphism group. If there is a central edge, then we preprocess the graph by inserting a dummy vertex into the central edge and two dummy edges, as shown in Fig. 6. Then apply the algorithm Cyclic, exactly as described above. Finally, remove the dummy vertex and edges. Because $k=2$, the central edge is drawn as a straight line after the removal of the dummy vertex and edges.

For the case $k=2$, we need to check correctness, as the argument above for the case $k \geq 3$ assumes that the central angle of the wedge is strictly less than $\pi$. For $k=2$, the concatenation $P_{12}$ of $P_{1}$ and $P_{2}$ forms a straight line, as in Fig. 7. The problem is that, although $P_{1}$ and $P_{2}$ are chordless, there may be a chord in $P_{12}$ from a vertex $u_{1}$ on $P_{1}$ to a vertex $u_{2}$ on $P_{2}$.

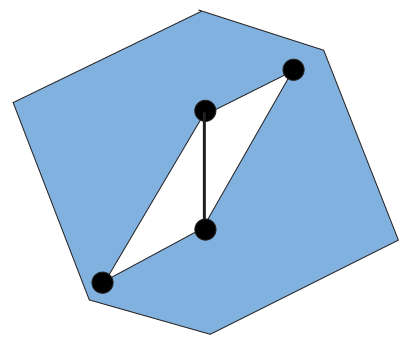

(a)

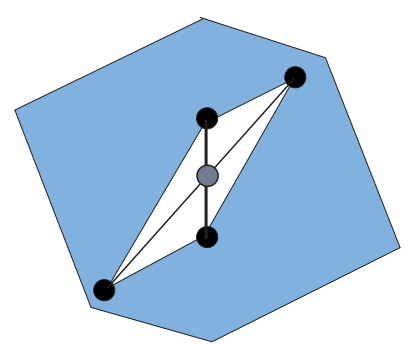

(b)

Fig. 6. Example of insertion of a dummy vertex and dummy edges for the case $k=2$. 


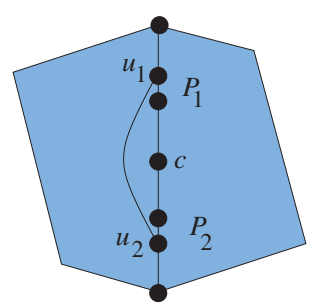

Fig. 7. Wedge for the case $k=2$.

However, we can show that the triconnectivity of $G$ implies that such a chord does not exist, as follows. Note that the rotational automorphism must map $u_{1}$ to $u_{2}$, that is, the edge $\left(u_{1}, u_{2}\right)$ is fixed. Also, the path $P_{12}$ is fixed. Thus every drawing of $G$ that displays the rotational symmetry is nonplanar (it must have the edge $\left(u_{1}, u_{2}\right)$ overlapping the path $P_{12}$ ). However, the Tutte drawing of $G$ in Section 4.1 is in fact planar and displays the rotational symmetry; we can conclude that $G$ is not triconnected, contrary to hypothesis.

Finally we note that each of these three steps of Algorithm Cyclic takes linear time.

Theorem 11. Algorithm Cyclic constructs a straight line drawing of a triconnected plane graph which shows $k$ rotational symmetries in linear time.

\subsection{One Axial Symmetry}

To display a single axial symmetry, we use a modification of Algorithm CYN.

A critical element of our modified Algorithm CYN concerns the subgraph that is fixed by the axial symmetry. Consider a drawing of a star-triangulated planar graph with one axial symmetry. There are fixed vertices, edges, and/or fixed faces on the axis; we need to characterize the subgraph formed by these.

We give some definitions. We define a diamond as either a triangle, or the 4-vertex graph in Fig. 8. The vertices of degree 3 in Fig. 8 are the middle vertices, and the edge joining them is the middle edge of the diamond.

A string of diamonds is a graph formed from a path $P=\left(v_{1}, v_{2}, \ldots, v_{\ell}\right), \ell \geq 2$, by a number (zero or greater) of "splitting" operations, as follows. If $1 \leq i \leq \ell-1$, then the edge $\left(v_{i}, v_{i+1}\right)$ may be replaced by a diamond as in Fig. 9(a). Alternatively, each of the end edges $\left(v_{1}, v_{2}\right)$ and $\left(v_{\ell-1}, v_{\ell}\right)$ may be replaced by a triangle, as in Fig. 9(b) and (c).

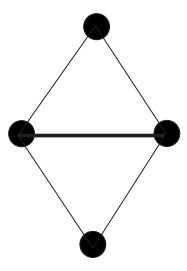

Fig. 8. Example of a diamond. 


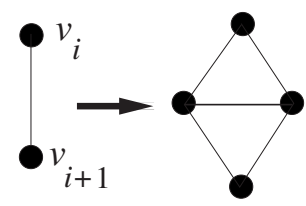

(a)

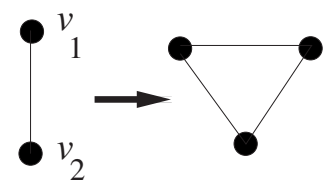

(b)

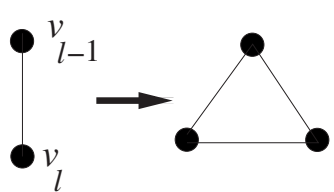

(c)

Fig. 9. Operations to form a string of diamonds.

Three strings of diamonds are illustrated in Fig. 10. Note that a string of diamonds is basically a path consisting of edges and diamonds; each end of the path may be a triangle.

Our interest in strings of diamonds is explained by the following lemma.

Lemma 6. Suppose that $\alpha$ is an axial automorphism of a star-triangulated planar graph $G$ whose set offaces is $F$. Let $V_{\mathrm{fix}}=\{v \in V \mid \alpha(v)=v\}, E_{\mathrm{fix}}=\{e \in E \mid \alpha(e)=$ $e\}$, and $F_{\mathrm{fix}}=\{f \in F \mid \alpha(f)=f\}$. The the subgraph of $G$ determined by $V_{\mathrm{fix}}, E_{\mathrm{fix}}$, and $F_{\mathrm{fix}}$ forms a string of diamonds that starts and finishes on the outside face.

Proof. The Tutte drawing $T$ of $G$ displays $\alpha$, from Theorem 9 . The axis of symmetry of $T$ cuts $G$ into a set of fixed vertices, triangular faces, and edges. It is easy to see that this set forms a string of diamonds.

The fixed string of diamonds is illustrated in Fig. 11.

To display a single axial symmetry, we need two steps. First we identify the fixed string of diamonds. Then we use Algorithm Symmetric_CYN, a modified version of Algorithm CYN. More formally, the algorithm is described below.
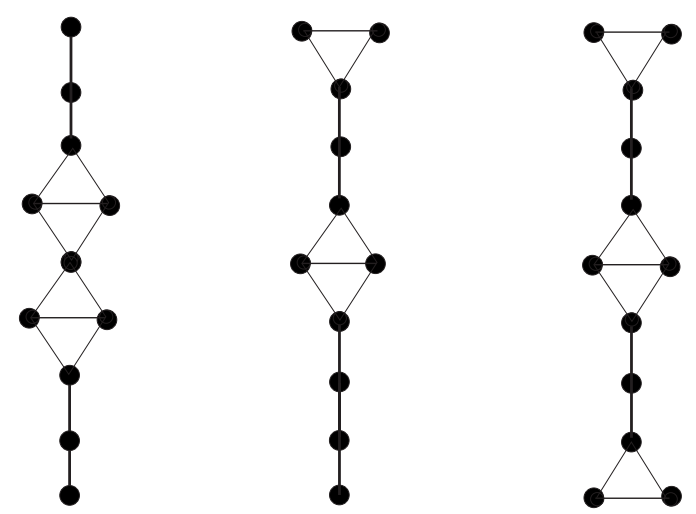

Fig. 10. Examples of strings of diamonds. 


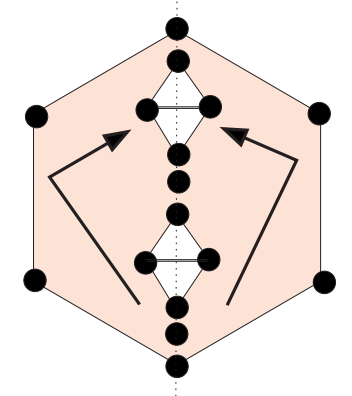

Fig. 11. Example of a fixed string of diamonds.

\section{Algorithm One_Axial}

1. Find a fixed string of diamonds. Suppose that $\omega_{1}, \omega_{2}, \ldots, \omega_{k}$ are the fixed edges and vertices in the fixed string of diamonds, in order from the outside face $\left(\omega_{1}\right.$ is on the outside face). For each $\ell, \omega_{\ell}$ may be a vertex or an edge. This is illustrated in Fig. 12.

2. Choose a symmetric convex polygon $S^{*}$ for the outside face $S$ of $G$.

3. Symmetric_CYN $\left(1, S^{*}, G, y_{1}\right)$, where $y_{1}$ denotes the position of $\omega_{1}$ on the axis of symmetry.

The main ingredient in Algorithm One_Axial is Algorithm Symmetric_CYN. To modify Algorithm CYN to display a single axial symmetry, we need to ensure that the

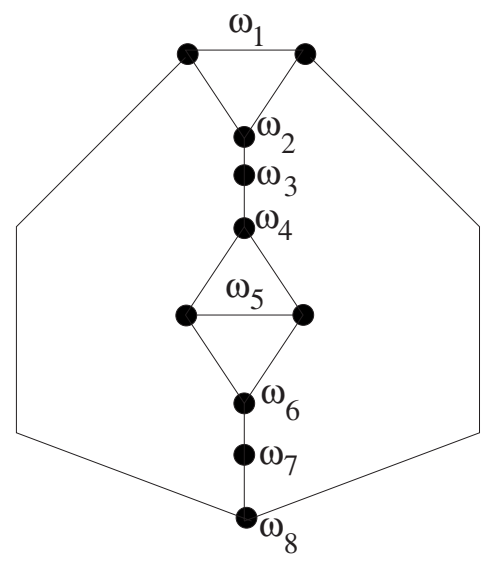

Fig. 12. Example of $\omega_{\ell}$. 


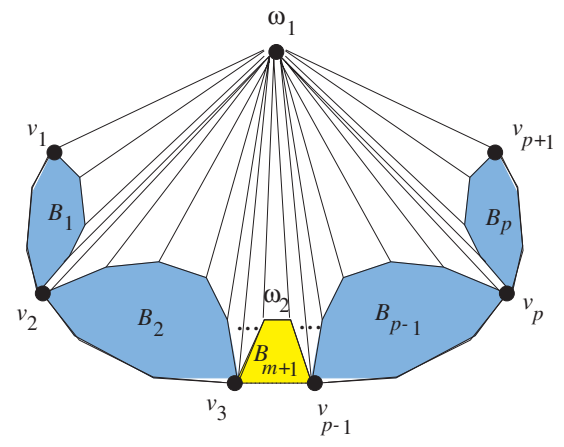

(a)

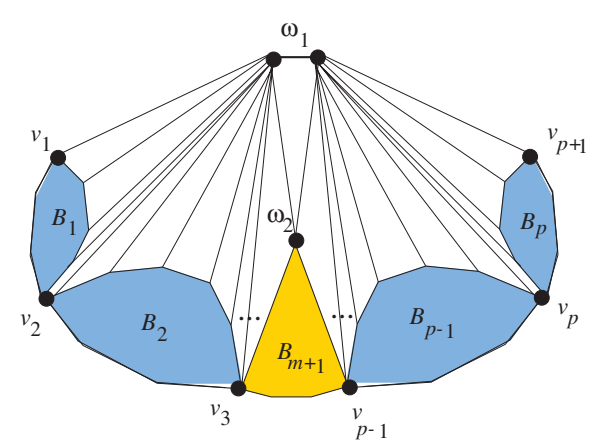

(b)

Fig. 13. Example of a symmetric version of CYN.

following three conditions are satisfied:

1. Choose $v$ in Step 1 of Algorithm CYN to be $\omega_{1}$, the first vertex or edge on the fixed string of diamonds. In Algorithm CYN, $v$ must be a vertex; here we extend Algorithm CYN to deal with an edge or a vertex. This condition is easy to satisfy; the two cases are illustrated in Fig. 13.

2. Let $D\left(B_{i}\right)$ be the drawing of $B_{i}$ and let $\alpha$ be the axial symmetry. Then $D\left(B_{i}\right)$ should be a reflection of $D\left(B_{j}\right)$, where $B_{j}=\alpha\left(B_{i}\right), i=1,2, \ldots, m$ and $m=\lfloor p / 2\rfloor$. To satisfy this condition, define $S_{j}^{*}$ to be the reflection of $S_{i}^{*}, i=1,2, \ldots, m$. Then we apply Algorithm CYN for $B_{i}, i=1,2, \ldots, m$, and construct $D\left(B_{j}\right)$ using a reflection of $D\left(B_{i}\right)$.

3. If $p$ is odd, then $D\left(B_{m+1}\right)$ should display axial symmetry. To satisfy this condition, we recursively apply Algorithm SymmetriC_CYN to $B_{m+1}$.

Note that the position of $\omega_{2}$ in Fig. 13 can be chosen arbitrarily along the axis of symmetry of $S^{*}$ within $S^{*}$. This means that we can specify the positions of the fixed vertices and middle edges along the axis of symmetry a priori, that is, as input to the algorithm. This is not strictly required here, but is helpful in Section 4.6.

We can now describe Algorithm Symmetri__CYN.

$$
\text { Algorithm SymmetriC_CYN }\left(\ell, S^{*}, G, y_{\ell}\right)
$$

Inputs:

$\ell:$ index of vertex or middle edge on the fixed string of diamonds

$S^{*}$ : a weakly convex polygon drawing of the outside face of $S$ of $G$

$G$ : a triangulated planar graph

$y_{\ell}:$ a position on the axis of symmetry for the fixed vertex or the fixed edge $\omega_{\ell}$

1. Delete $\omega_{\ell}$ from $G$ together with edges incident to $\omega_{\ell}$. Divide the resulting graph $G^{\prime}=G-\omega_{\ell}$ into the blocks $B_{1}, B_{2}, \ldots, B_{p}, p \geq 1$, ordered anticlockwise around the outside face. Let $m=\lfloor p / 2\rfloor$.

2. Determine a convex polygon $S_{i}^{*}$ of the outside facial cycle $S_{i}$ of each $B_{i}$ so that $B_{i}$ with $S_{i}^{*}$ satisfy the conditions in Theorem 10 and $S_{p-i+1}^{*}$ is a reflection of $S_{i}^{*}$. 
3. For each $i=1$ to $m$ :

(a) Construct a drawing $D\left(B_{i}\right)$ of $B_{i}$ using Algorithm CYN.

(b) Construct $D\left(B_{p-i+1}\right)$ as a reflection of $D\left(B_{i}\right)$.

4. If $p$ is odd, then construct a drawing $D\left(B_{m+1}\right)$ using Symmetric_CYN $(\ell+$ $\left.1, S_{m+1}^{*}, B_{m+1}, y_{\ell+1}\right)$.

5. Merge the $D\left(B_{i}\right)$ to form a drawing of $G$, placing $\omega_{\ell}$ at $y_{\ell}$.

Using the same argument as for the linearity of Algorithm CYN [7], one can show that Algorithm One_Axial takes linear time.

Theorem 12. Algorithm One_Axial constructs a straight line drawing of a triconnected plane graph which shows one axial symmetry in linear time.

\subsection{The Dihedral Case}

In this section we give an algorithm for displaying a dihedral group $\langle\rho, \alpha\rangle$, where $\rho$ is a rotation of order $k$ and $\alpha$ is an axial automorphism. We assume that $\rho$ is displayed as a clockwise rotation.

As with the cyclic case, there is either a central vertex or a central edge (after the star triangulation) and the central edge case can be transformed to the central vertex case in the same way as for the cyclic case in Section 4.4. Thus we assume that there is a central vertex.

We adopt the same general strategy as for the cyclic case: we divide the graph into "wedges", draw each wedge, and then merge the drawings of wedges together to make a drawing of the whole graph. However, the dihedral case is more difficult than the pure rotational case, because an axial symmetry in the dihedral group can have fixed faces as well as fixed edges. This means that the boundary of a wedge may be a fixed string of diamonds as in the one axial case, rather than a simple path. To achieve dihedral symmetry, the axis of symmetry must be the perpendicular bisector of the middle edge of each diamond. This makes the merging operation more difficult.

Consider a drawing of a triconnected planar graph with a dihedral symmetry group of size $2 k$. There are $k$ axial symmetries, with axes at angles of $\pi i / k, 0 \leq i \leq k-1$, to the $x$ axis, as in Fig. 14(a). Roughly speaking, a wedge is the area between two adjacent axes, as in Fig. 14(b). Note that in these wedges, the boundaries $P_{1}$ and $P_{2}$ may be strings of diamonds. These may terminate in a triangle.

Now we describe the symmetric drawing algorithm for the dihedral case. As with the cyclic case, we have three steps.

\footnotetext{
Algorithm Dihedral

1. Find_Wedge_Dihedral.

2. Draw_Wedge_Dihedral.

3. Merge_Wedges_Dihedral.
}

Now we describe each of these three steps. 


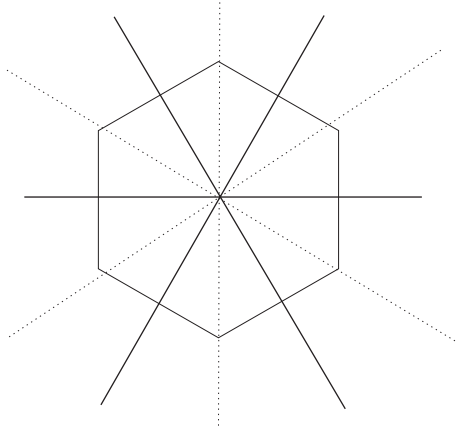

(a)

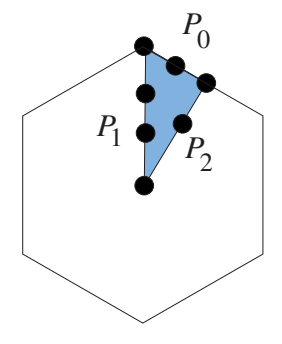

(b)

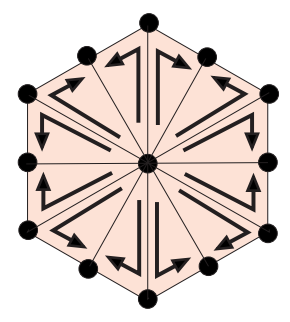

(c)

Fig. 14. Wedge for the dihedral case.

The first step is to define the "wedge" subgraph precisely. This is defined in a different way from the cyclic case. We define a wedge by finding two fixed strings of diamonds.

\section{Algorithm Find_Wedge_Dihedral}

1. Find the central vertex $c$.

2. Find a string of diamonds $P_{1}$ that is fixed by $\alpha$, from $c$ to a vertex $v$ or an edge $e$ on the outside face.

3. Traverse the outside face, clockwise from $v$ (or $e$ ) to the vertex $v^{\prime}$ or edge $e^{\prime}$ that is fixed by $\rho^{-1} \alpha \rho$. Let $P_{0}$ denote the path so traversed.

4. Find the string of diamonds $P_{2}$ for $\rho^{-1} \alpha \rho$, from $c$ to $v^{\prime}$ (or $e^{\prime}$ ).

5. Define the wedge $W$ to be the subgraph enclosed by $P_{0}, P_{1}$, and $P_{2}$, including the vertices and edges of $P_{0}, P_{1}$, and $P_{2}$.

Note that one can find $c$ and the two fixed strings of diamonds $P_{1}$ and $P_{2}$ in linear time using the generators of the group.

The second step, Draw_Wedge_Dihedral, constructs a drawing of the wedge. As mentioned above, this step must ensure that the middle edge of each diamond on the boundary is orthogonal to the axis of reflection.

We need further terminology for diamonds. The upper vertex is a vertex of a diamond that is farthermost from the fixed centre vertex. Similarly we can define the lower vertex of a diamond.

The input to Draw_Wedge_Dihedral is a plane graph $W$ and a corresponding polygon $C$ with triangle shape, as in Fig. 14(b). The boundary of the outside face of $W$ consists of the outside path $P_{0}$ and two fixed strings of diamonds $P_{1}$ and $P_{2}$.

We must consider some special case where the diamonds on the boundary of $W$ can intersect. This can occur in several ways. In Fig. 15, $P_{1}$ and $P_{2}$ have two diamonds which share a vertex; we call these kissing diamonds.

In Fig. 16, $P_{1}$ and $P_{2}$ have diamonds which share an edge; we call these overlapping diamonds. 


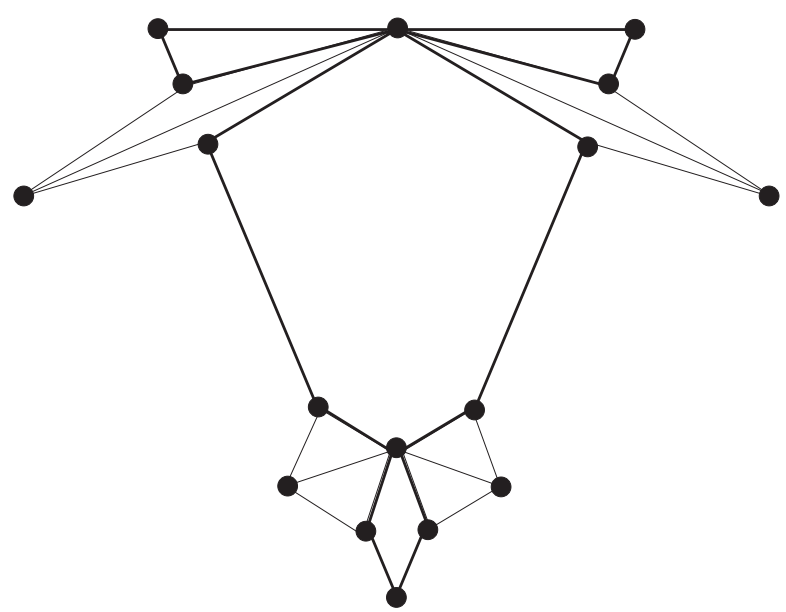

Fig. 15. Example of kissing diamonds.

A diamond on boundary $P_{i}$ is special if one of its middle vertices lies on $P_{2-i+1}$, for $i=1,2$, as in Fig. 17. Figure 17 shows other special diamonds; $P_{1}$ and $P_{2}$ have diamonds which share a vertex. We also include kissing and overlapping diamonds as special diamonds.

Roughly speaking, the algorithm Draw_Wedge_Dihedral runs as follows. First we find all the special diamonds and we draw them. Then we choose the positions of all the fixed vertices of $P_{1}$ and $P_{2}$ that have not been drawn so far. Next we subdivide the wedge in various ways to form "subwedges". Finally we draw each of these subwedges.

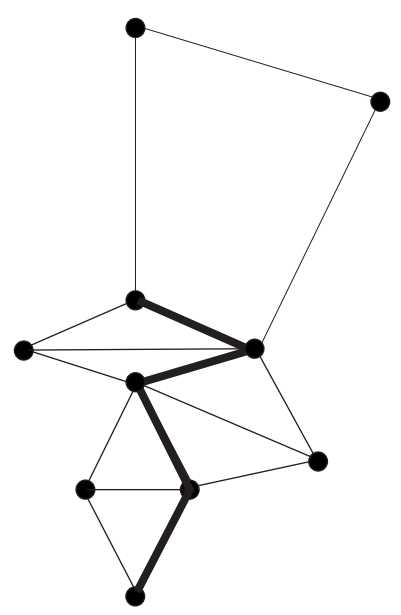

Fig. 16. Example of overlapping diamonds. 


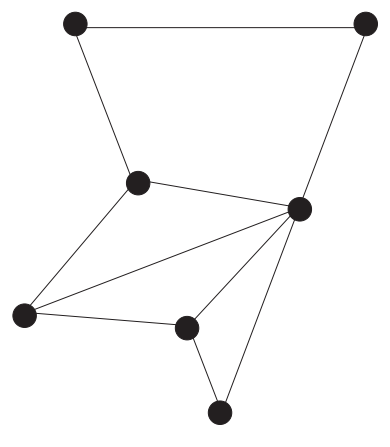

(a)

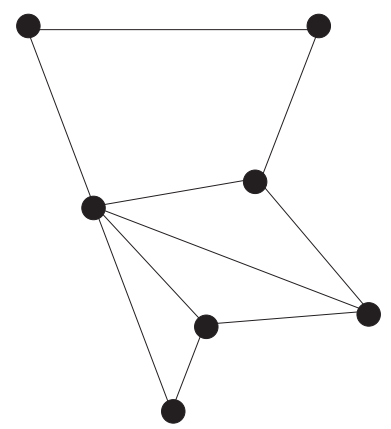

(b)

Fig. 17. Other special diamonds.

Algorithm Draw_Wedge_Dihedral

1. If there are special diamonds, then Draw_Special_Diamonds. Note that this divides the wedge $W$ into subgraphs called subwedges.

2. Choose a position for each of the fixed vertices on $P_{1}$ and $P_{2}$ that have not been drawn so far. These are the upper and lower vertices of non-special diamonds, as well as the fixed vertices that are not in diamonds. For each non-special diamond on $P_{1}$ and $P_{2}$, we also choose a position for the midpoint of the middle edge.

3 . If there are "wedge-chords" (defined below), then further subdivide each subwedge using wedge-chords.

4. Draw each subwedge $W_{i}$ using Algorithm Draw_Subwedge.

First we describe Algorithm Draw_Special_Diamonds. For each of $P_{1}$ and $P_{2}$, the algorithm numbers the vertices from the centre vertex to the outside face. It then labels all the special diamonds (on both $P_{1}$ and $P_{2}$ ) with positive integers in a way that is consistent (in a sense defined below) with both the order on $P_{1}$ and the order on $P_{2}$. Using these labels, the algorithm computes the radius of each special diamond.

\section{Algorithm Draw_Special_Diamonds}

1. For $i=1,2$, number the vertices on $P_{i}$ with positive integers increasing from the central vertex outward. The two middle vertices of a diamond receive the same number.

2. Label all the special diamonds with positive integers such that the following properties hold:

- The order of the labels on the special diamonds is consistent with numbering of the vertices of the diamonds. That is, if a diamond labelled $w$ contains a vertex numbered $y$ on $P_{i}$ and a diamond labelled $x$ contains a vertex numbered $z$ on $P_{i}$, then $y \leq z$ implies that $w \leq x$.

- A pair of kissing diamonds are labelled with the same integer. 


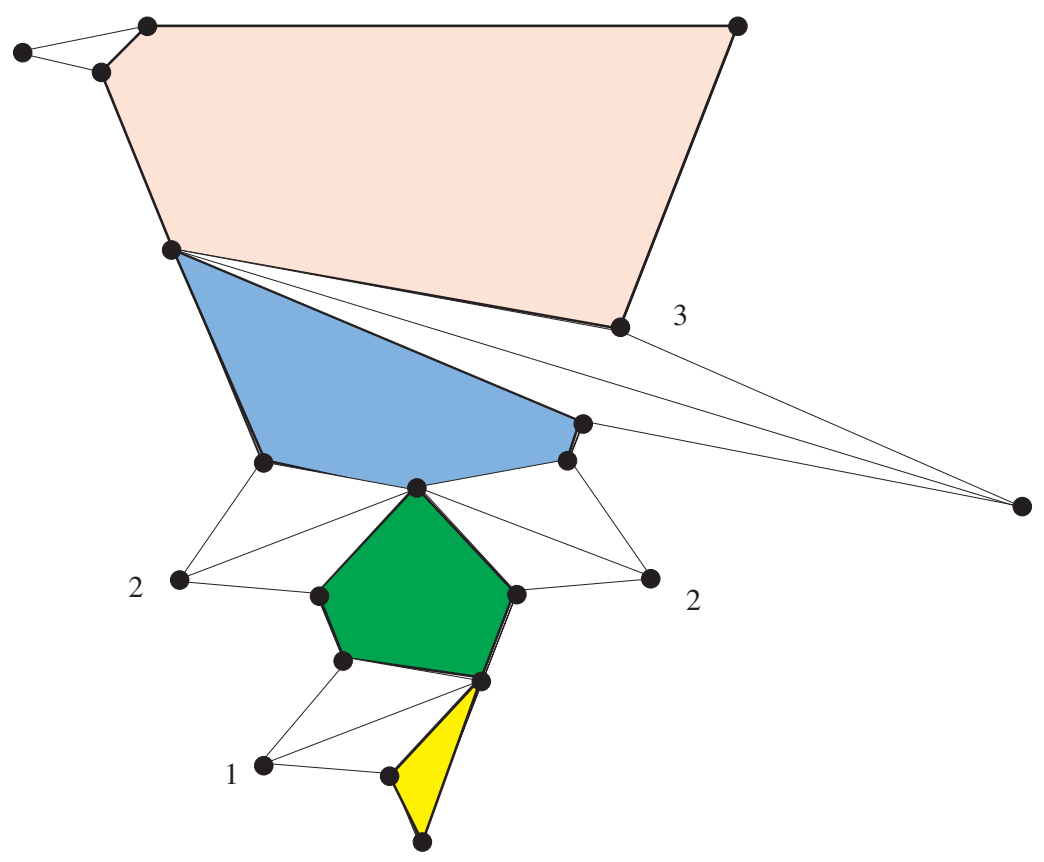

Fig. 18. Example of labels for special diamonds.

- If a middle vertex of a diamond labelled $x$ is the lower vertex of a diamond labelled $y$, then $y=x+1$.

An example of labels of diamonds is illustrated in Fig. 18.

3. For each $i$, place the midpoint of the middle edge of special diamond $i$ at radius $3^{i}$. (Note that because these diamonds are special, the positions of the middle vertices are forced by the position of the midpoint of the middle edge.)

4. For each $i$, if the upper vertex of special diamond $i$ has not yet been placed, then place it at radius $\frac{5}{2} 3^{i}$, unless it is a middle vertex of special diamond $i+1$; in which case the upper vertex is forced at radius $3^{i+1} / \sin \theta$, where $\theta$ is the wedge angle. (Note this cannot happen for $\theta=\pi / 2$.)

5 . For each $i$, if the lower vertex of special diamond $i$ has not yet been placed, then place it at radius $3^{i}-1$.

An example of placing special diamonds is illustrated in Fig. 19.

Lemma 7. Algorithm Draw_Special_Diamonds places all special diamonds with no edge intersections, such that every internal face of the resulting drawing is strictly convex.

Proof. Note that drawing the overlapping diamonds introduces chords in $C$ and divides $C$ into convex subpolygons. Also, it is easy to check that the placement of kissing diamonds defined in Algorithm Draw_Special_Diamonds introduces convex angles at the kissing point. 


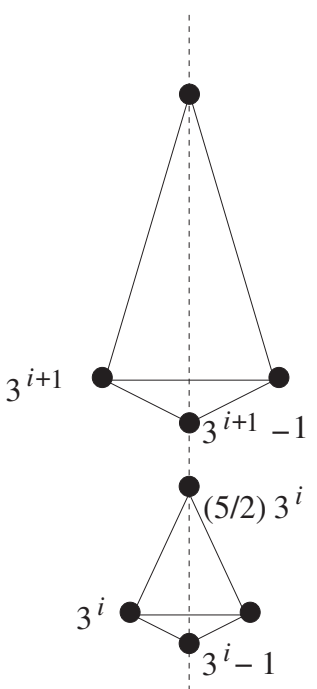

Fig. 19. Example of placing special diamonds.

The next step of Algorithm Draw_Wedge_Dihedral is to choose positions for the parts of $P_{1}$ and $P_{2}$ that have not been drawn so far, including the midpoints of the non-special diamonds. This can be done by spacing them equally between the special diamonds. Note that although we have chosen positions for the midpoints of the middle edges of non-special diamonds, we have not chosen the positions for the middle vertices of non-special diamonds.

At this stage we need to draw subwedges in the convex internal faces mentioned in Lemma 7, formed after the special diamonds have been drawn. This is done using Algorithm Draw_Subwedge, which we describe below. However, before we proceed, we must further subdivide the subwedges. The problem is that the subwedge may have chords, as in Fig. 20. For reasons that become clear below, we need to subdivide subwedges with these chords.

First we need to consider chords that cross from $P_{i}$ to $P_{j}$, where $j \neq i, i=0,1,2$. A wedge-chord is an edge $(u, v)$ such that $u$ is on $P_{i}$ and $v$ is on $P_{j}, j \neq i, i=0,1,2$. All the wedge-chords can be found in linear time.

Wedge-chords further divide the subwedges into $W_{1}, W_{2}, \ldots, W_{m}$. If we draw the wedge-chords, then we form corresponding convex polygons $C_{1}, C_{2}, \ldots, C_{m}$.

As long as we choose the length of the middle edge of each non-special diamond to be small enough, we can ensure that each $C_{i}$ is convex. Figure 20 shows examples of wedge-chords and subwedges.

By subdividing subwedges along wedge-chords, we can assume that the input graph $W_{i}$ of Draw_Subwedge has no wedge-chords. This is important because of the following technical lemma.

Lemma 8. Suppose that $W_{i}$ is a triangulated plane graph with outside face $S$ such that $S$ has no chords. Suppose that $B$ is a set of vertices on $S$ (not necessarily every vertex of $S)$. Then $W_{i}^{\prime}=W_{i}-B$ is connected. 


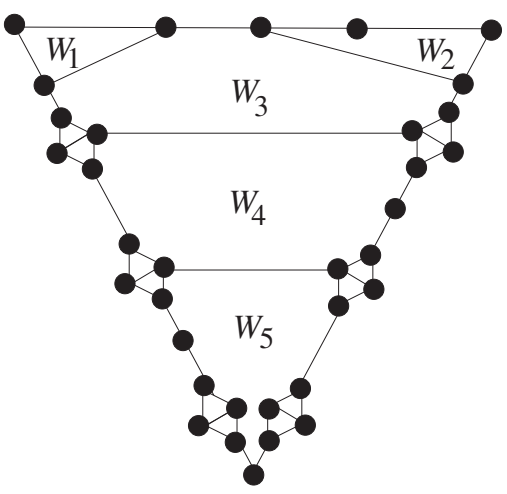

(a)

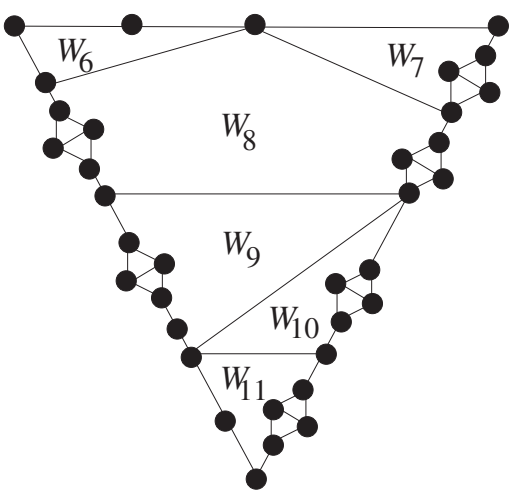

(b)

Fig. 20. Examples of wedge-chords and subwedges.

Proof. Firstly we show that $W_{i}$ is triconnected. It is well known that every simple planar triangulation (the outside face being also a triangle) is triconnected. Form a simple planar triangulation $W_{i}^{+}$from $W_{i}$ by star triangulating the outside face of $W_{i}$ using a new vertex $x$. This gives a simple triangulation, which must be triconnected. The only way that removing a vertex from $W_{i}^{+}$reduces the connectivity of a triconnected graph is when the vertex is in a cut of size 3 . Such a cut through $x$ is a separating cycle of length 3 , which obviously needs to use a chord of the outside face of $W_{i}$. Since $W_{i}$ is chordless, we can conclude that it is triconnected.

Suppose that $W_{i}^{\prime}$ is not connected, and let $u$ and $v$ be vertices in different components of $W_{i}^{\prime}$. Since $W_{i}$ is triconnected, there are three vertex-disjoint paths $\pi_{1}, \pi_{2}$, and $\pi_{3}$ between $u$ and $v$. Since $u$ and $v$ are in different components of $W_{i}^{\prime}$, each of these paths must contain a vertex of $B$; suppose that $\pi_{j}$ contains $w_{j} \in B$ for $j=1,2,3$. Now consider the six vertices $u, v, x, w_{1}, w_{2}, w_{3}$ in $W_{i}^{+}$. The paths $\pi_{1}, \pi_{2}$, and $\pi_{3}$ together with the edges $\left(x, w_{j}\right), j=1,2,3$, form a subgraph of $W_{i}^{+}$that is homomorphic to $K_{3,3}$ (see Fig. 21), contradicting the planarity of $W_{i}^{+}$. We can conclude that $W_{i}^{\prime}$ is connected.

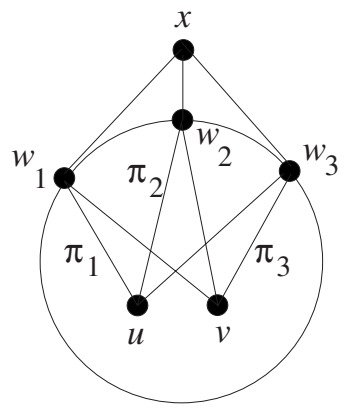

Fig. 21. $K_{3,3}$ in $W_{i}^{+}$. 
The input to Draw_Subwedge is somewhat similar to the input of Draw_Wedge_ Dihedral. We have a plane graph $W_{i}$ and a convex polygon $C_{i}$. The boundary of the outside face of $W_{i}$ consists of two fixed strings of diamonds $P_{1}$ and $P_{2}$ and a number of simple paths. These strings of diamonds and paths are assigned to sides of $C_{i}$. We aim to draw $W_{i}$ in $C_{i}$ such that the strings of diamonds and the simple paths lie on their assigned paths. Further, the side assigned to $P_{i}$ must be the perpendicular bisector of the middle edge of each diamond on $P_{i}$, for $i=1,2$.

We now describe Algorithm Draw_Subwedge. The input is a graph $W_{i}$ and a convex polygon $C_{i}$; there are no special diamonds and no wedge-chords. Note that at most two sides, $P_{1}$ and $P_{2}$, have diamonds. The algorithm can be divided into three cases, depending on whether $P_{1}$ and $P_{2}$ contain diamonds:

1. Both $P_{1}$ and $P_{2}$ have no diamonds.

2. Only one of $P_{1}$ and $P_{2}$ has diamonds.

3. Both $P_{1}$ and $P_{2}$ have diamonds.

We now treat these cases in turn below.

The first case is where there is no diamond in $P_{1}$ and $P_{2}$. Here we can use the same drawing algorithm as in the cyclic case, CYN.

The second case is where only one of $P_{1}$ and $P_{2}$ has diamonds; suppose that it is $P_{1}$. Note that $P_{1}$ is the border of two wedges. The union of these two wedges is a wedge which has an axial planar automorphism, and each side is a simple path with no diamonds. This can be drawn with axial symmetry using SymmetriC_CYN.

For the third case we use Lemma 8 to divide the subwedge into two. The application of Lemma 8 requires some care. First, for $i=1,2$, let $P_{i}^{\prime}$ be the path from the start vertex $u_{i}$ to the finish vertex $v_{i}$ of $P_{i}$ that consists of all the fixed vertices on $P_{i}$ plus the middle vertices that are not on the outside face of the wedge. This is illustrated in Fig. 22.

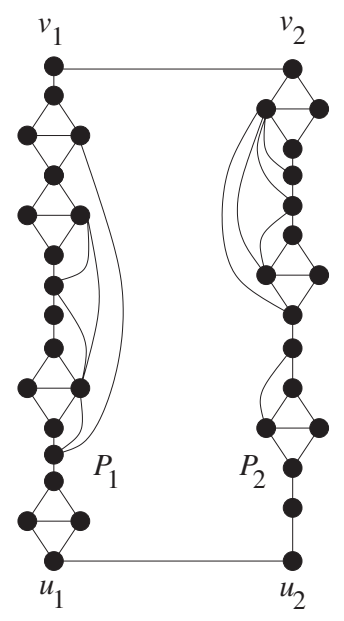

(a)

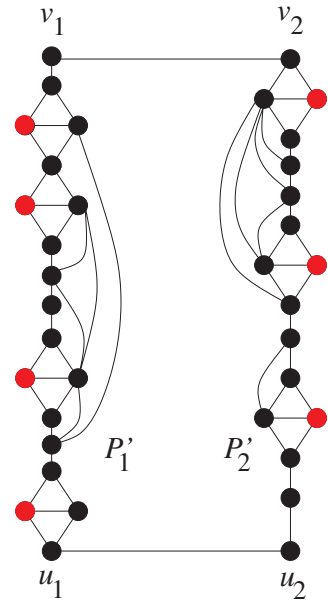

(b)

Fig. 22. Paths $P_{i}$ and $P_{i}^{\prime}$. 


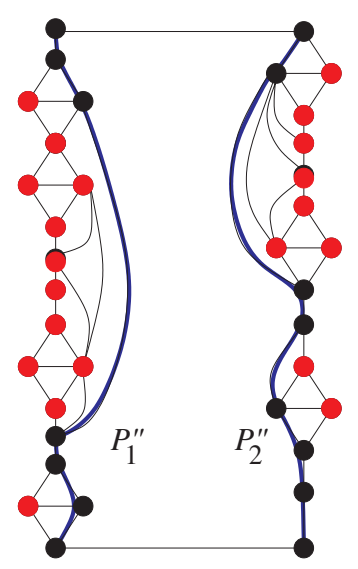

Fig. 23. Paths $P_{i}^{\prime \prime}$.

Now we impose a partial order on the chords and edges of $P_{i}^{\prime}=\left(a_{1}, a_{2}, \ldots, a_{m}\right)$ as follows. If $\left(a_{q}, a_{r}\right)$ and $\left(a_{s}, a_{t}\right)$ are two chords or edges of $P_{i}^{\prime}$ then $\left(a_{q}, a_{r}\right) \leq\left(a_{s}, a_{t}\right)$ if and only if $s \leq q$ and $r \leq t$. Intuitively, $\left(a_{q}, a_{r}\right) \leq\left(a_{s}, a_{t}\right)$ when $\left(a_{q}, a_{r}\right)$ is "inside" $\left(a_{s}, a_{t}\right)$, as illustrated in Fig. 22(b).

Now consider the path $P_{i}^{\prime \prime}$ from $u_{i}$ to $v_{i}$ consisting only of maximal (in the partial order) edges and chords of $P_{i}^{\prime}$, as in Fig. 23. Clearly $P_{i}^{\prime \prime}$ is chordless.

To apply Lemma 8 , we let $W_{i}$ be the subgraph of the wedge bounded by $P_{1}^{\prime \prime}$ and $P_{2}^{\prime \prime}$. Since there are no wedge-chords, and $P_{1}^{\prime \prime}$ and $P_{2}^{\prime \prime}$ are chordless, the outside face of $W_{i}$ is chordless. Let $B$ be the set of every vertex on $P_{1}^{\prime \prime}$ and $P_{2}^{\prime \prime}$ except $u_{1}$ and $v_{2}$. From Lemma 8, the subgraph $W_{i}-B$ is connected, and so contains a chordless (for example, shortest) path from $u_{2}$ to $v_{1}$; see Fig. 24(a). This chordless path can be used to divide the subwedge into two subwedges, each of which has at most one string of diamonds on the boundary. For each of these two subwedges, we can use Symmetric_CYN in the same way as for the second case; see Fig. 24(b).

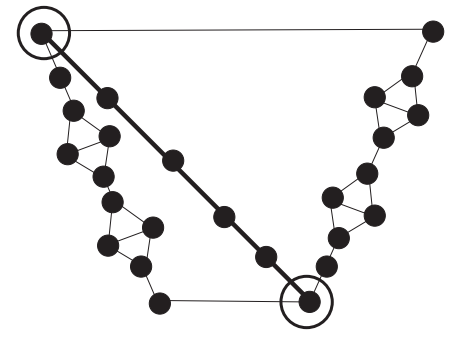

(a)

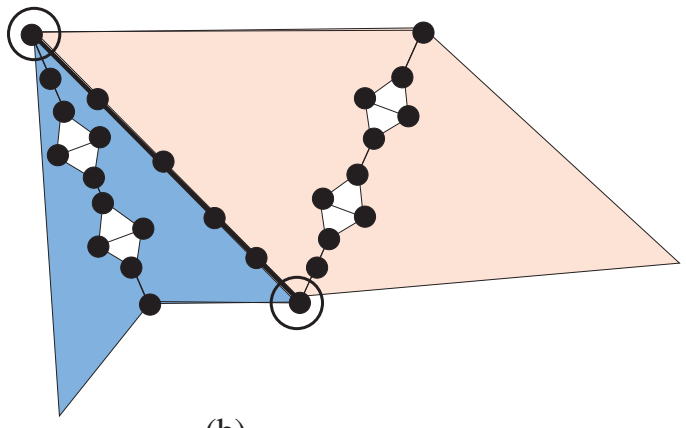

(b)

Fig. 24. (a) Chordless path from $u_{2}$ to $v_{1}$, and (b) applying SymmetriC_CYN. 
We now describe Algorithm Draw_Subwedge, which draws a subwedge $W_{i}$.

$$
\text { Algorithm Draw_Subwedge }\left(W_{i}\right)
$$

Input:

$W_{i}$ : a subwedge

1. If there are no diamonds on both $P_{1}$ and $P_{2}$, then draw $W_{i}$ using CYN.

2. If only one of $P_{1}$ and $P_{2}$ has diamonds, then

(a) Define a wedge $W W$ which has one axial symmetry using two copies of $W_{i}$.

(b) Draw $W W$ using Symmetric_CYN. else

(a) Find a chordless path from the start vertex of $P_{2}$ to the finish vertex of $P_{1}$ that does not intersect $P_{1}$ or $P_{2}$, as in Fig. 24(a).

(b) Divide $W_{i}$ into two regions $W_{1}$ and $W_{2}$ using the chordless path.

(c) Draw $W_{1}$ and $W_{2}$ respectively using Symmetri__CYN as in Fig. 24(b).

To complete Algorithm Dihedral, we must describe Algorithm Merge_Wedges_ Dihedral. This algorithm simply constructs a drawing for the whole graph by replicating the drawing $D$ of the wedge $W$. The details of this step differ according to the three different cases in the previous step. We briefly describe the merge step for the first case. To display the axial symmetries, we construct a drawing $D^{\prime}$, which is a reflection of the drawing $D$ of the wedge $W$ in an axis. Then we make $k$ copies each of $D$ and $D^{\prime}$. Finally we arrange them in a way to display rotations as well as reflections to construct a drawing for the whole graph. An example is illustrated in Fig. 25.

Clearly each step of Algorithm Dihedral takes linear time. We now summarize the performance of Algorithm Dihedral in the following theorem.

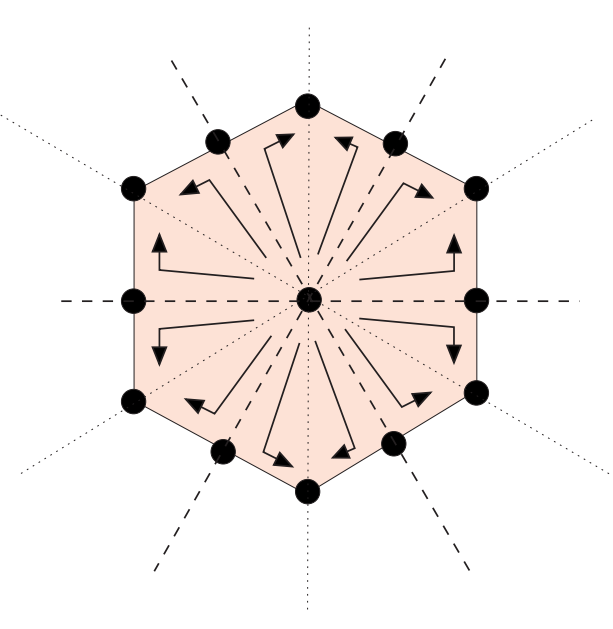

Fig. 25. Example of merging process. 
Theorem 13. Algorithm Dihedral constructs a straight line drawing of a triconnected plane graph which shows $k$ rotational symmetries and $k$ axial symmetries in linear time.

\section{Conclusion}

In this paper we show that one can construct a maximally symmetric drawing of triconnected planar graphs with straight lines in linear time. We present two algorithms. First we give a linear time algorithm for finding a maximum size planar automorphism group. Next we present a linear time symmetric drawing algorithm with straight line edges.

It is possible to extend this work into the case of biconnected planar graphs with a given topological embedding in the plane, as follows. Given the embedding, we apply star triangulation to make it triconnected. Lemma 2 can be extended to ensure that this process does not lose planar automorphisms. The drawing algorithm proceeds exactly as in Section 4.

The extension to planar graphs for which the embedding is not prescribed is much more difficult; see [15], [17], and [18].

\section{References}

1. D. Abelson, S. Hong and D. E. Taylor, A Group-Theoretic Method for Drawing Graphs Symmetrically, Graph Drawing, Proc. of GD 2002, Lecture Notes in Computer Science 2528, pp. 86-97, Springer-Verlag, Berlin, 2002.

2. M. A. Armstrong, Groups and Symmetry, Springer-Verlag, New York, 1988.

3. L. Babai, Automorphism Groups, Isomorphism, and Reconstruction, in Handbook of Combinatorics, Volume 2 (Eds. R. L. Graham, M. Groetschel and L. Lovasz), Chapter 27, Elsevier Science, Amsterdam, 1995.

4. M. Bern and D. Eppstein, Optimal Mobius Transformations for Information Visualization and Meshing, Proc. of WADS 2001, Lecture Notes in Computer Science 2125, pp. 14-25, Springer-Verlag, Berlin, 2001.

5. J. A. Bondy and U. S. R. Murty, Graph Theory with Applications, North-Holland, Amsterdam, 1976.

6. C. Buchheim and M. Junger, Detecting Symmetries by Branch and Cut, Graph Drawing, Proc. of GD 2001, Lecture Notes in Computer Science 2265, pp. 178-188, Springer-Verlag, Berlin, 2001.

7. N. Chiba, T. Yamanouchi and T. Nishizeki, Linear Algorithms for Convex Drawings of Planar Graphs, Progress in Graph Theory, pp. 153-173, Academic Press, New York, 1984.

8. G. Di Battista, P. Eades, R. Tamassia and I. G. Tollis, Graph Drawing: Algorithms for the Visualization of Graphs, Prentice-Hall, Englewood Cliffs, 1998.

9. P. Eades and X. Lin, Spring Algorithms and Symmetry, Theoretical Computer Science, 240(2) (2000), 379-405.

10. M. Fontet, Linear Algorithms for Testing Isomorphism of Planar Graphs, Proc. Third Colloquium on Automata, Languages, and Programming, pp. 411-423, 1976.

11. H. de Fraysseix, A Heuristic for Graph Symmetry Detection, Graph Drawing, Proc. of GD 1999, Lecture Notes in Computer Science 1731, pp. 276-285, Springer-Verlag, Berlin, 1999.

12. S. Hong, Drawing Graphs Symmetrically in Three Dimensions, Graph Drawing, Proc. of GD 2001, Lecture Notes in Computer Science 2265, pp. 189-204, Springer-Verlag, Berlin, 2002.

13. S. Hong and P. Eades, An Algorithm for Finding Three Dimensional Symmetry in Trees, Graph Drawing, Proc. of GD 2000, Lecture Notes in Computer Science 1984, pp. 360-371, Springer-Verlag, Berlin, 2000.

14. S. Hong and P. Eades, An Algorithm for Finding Three Dimensional Symmetry in Series Parallel Digraphs, Algorithms and Computation, Proc. of ISAAC 2000, Lecture Notes in Computer Science 1969, pp. 266277, Springer-Verlag, 2000. 
15. S. Hong and P. Eades, Symmetric Layout of Disconnected Graphs, Algorithms and Computation, Proc. of ISAAC 2003, Lecture Notes in Computer Science 2906, pp. 405-414, Springer-Verlag, Berlin, 2003.

16. S. Hong and P. Eades, Drawing Trees Symmetrically in Three Dimensions, Algorithmica, 36(2) (2003), 153-178.

17. S. Hong and P. Eades, Drawing Planar Graphs Symmetrically II: Biconnected Planar Graphs, Algorithmica, 42(2) (2005), 159-197.

18. S. Hong and P. Eades, Drawing Planar Graphs Symmetrically III: Oneconnected Planar Graphs, Algorithmica, 44(1) (2006), 67-100.

19. S. Hong, P. Eades and S. Lee, An Algorithm for Finding Geometric Automorphisms in Planar Graphs, Algorithms and Computation, Proc. of ISAAC 1998, Lecture Notes in Computer Science 1533, pp. 277286, Springer-Verlag, Berlin, 1998.

20. S. Hong, P. Eades and S. Lee, Drawing Series Parallel Digraphs Symmetrically, Computational Geometry: Theory and Applicatons, 17(3-4) (2000), 165-188.

21. S. Hong, P. Eades, A. Quigley and S. Lee, Drawing Algorithms for Series-Parallel Digraphs in Two and Three Dimensions, Graph Drawing, Proc. of GD 1998, Lecture Notes in Computer Science 1547, pp. 198-209, Springer-Verlag, Berlin, 1998.

22. S. Hong, B. McKay and P. Eades, Symmetric Drawings of Triconnected Planar Graphs, Proc. of SODA 2002, pp. 356-365, 2002.

23. X. Lin, Analysis of Algorithms for Drawing Graphs, Ph.D. Thesis, University of Queensland, 1992

24. R. J. Lipton, S. C. North and J. S. Sandberg, A Method for Drawing Graphs, in Proc. of ACM Symposium on Computational Geometry, pp. 153-160, ACM, New York, 1985.

25. A. Lubiw, Some NP-Complete Problems Similar to Graph Isomorphism, SIAM Journal on Computing, 10(1) (1981), 11-21.

26. P. Mani, Automorphismen von Polyedrischen Graphen, Mathematische Annalen, 192 (1971), 279-303.

27. J. Manning, Geometric Symmetry in Graphs, Ph.D. Thesis, Purdue University, 1990.

28. J. Manning and M. J. Atallah, Fast Detection and Display of Symmetry in Trees, Congressus Numerantium, 64 (1988), 159-169.

29. J. Manning and M. J. Atallah, Fast Detection and Display of Symmetry in Outerplanar Graphs, Discrete Applied Mathematics, 39 (1992), 13-35.

30. H. Purchase, Which Aesthetic Has the Greatest Effect on Human Understanding?, Graph Drawing, Proc. of GD '97, Lecture Notes in Computer Science 1353, pp. 248-259, Springer-Verlag, Berlin, 1998.

31. C. Thomassen, Planarity and Duality of Finite and Infinite Graphs, Journal of Combinatorial Theory, Series B, 29 (1980), 244-271.

32. W. T. Tutte, Convex Representations of Graphs, Proceedings of the London Mathematical Society, 10(3) (1960), 304-320.

33. W. T. Tutte, How to Draw a Graph, Proceedings of the London Mathematical Society, 13 (1963), 743-768.

Received November 7, 2004, and in revised form April 29, 2005. Online publication July 13, 2006. 